International Journal of Engineering \& Technology, $7(4.11)(2018) 212-216$
International Journal of Engineering \& Technology
SPC

\title{
Performance Analysis of LPWAN Using LoRa Technology for IoT Application
}

\author{
Amir Muaz Abdul Rahman, Fadhlan Hafizhelmi Kamaru Zaman*, Syahrul Afzal Che Abdullah \\ Faculty of Electrical Engineering, University Teknologi MARA (UiTM), 40450 Shah Alam, Selangor, Malaysia \\ *Corresponding author email: fadhlan@salam.uitm.edu.my
}

\begin{abstract}
This paper was dedicated to study the performance of an Internet of Things (IoT) application using LoRa Wide Area Network (LoRaWAN). LoRa is a Low Power Wide Area Network (LPWAN) technology developed for IoT applications specifically. Due to the facts that LoRa is a new product, there are questions about its reliability. Hence, a conclusive experiment has been made. The experiment conducted to get an insight to LoRa received signal strength (RSSI) and packet loss. The analysis also includes a measurement of the application Signal to Noise Ratio (SNR) between the transmitter and receiver. The results of the experiment show that with a higher spreading factor, LoRa end device provides more immunity against multi-path and signal fading. The proposed IoT application based on this LoRa technology is for autonomous vehicle status information transmission and intervehicle communications, specifically deployed in UiTM Autonomous Vehicle 1 (UiTM AV1).
\end{abstract}

Keywords: Internet of Things; LoRa; LPWAN; outdoor; performance analysis.

\section{Introduction}

In this time of the centuries, literally, everything is connected to the internet ranging from phones, computers, home appliances, vehicles and many other electronics devices. This created a network that's called "Internet of Things" (IoT) is defined as a network system where devices or applications are interconnected to each other with the presence of the Internet. Each of these devices or applications is unique to their own and can only be identified through their internal computing system that connected to the network infrastructures [1].

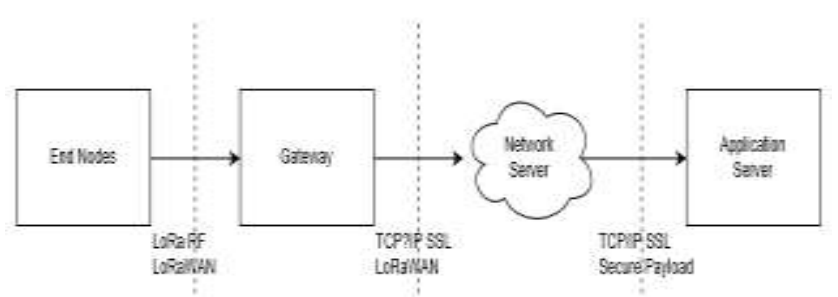

Fig. 1: General LoRaWAN Network Topology

Typically, a LoRaWAN system consists of a sensor node connected to the IoT system through a gateway. The node is a transducer that giving out an appropriate output in electrical signal based on events or even changes in quantity [2]. The gateway is basically a medium to connect the nodes to the internet and the network server. From there, the data will then send to the application server. To date, various LPWAN based technology such as WAVIoT Narrowband M2M protocol, Ultra Narrow Band (UNB), and LoRaWAN have coexist together [3]. LPWAN technologies are using a relatively low frequency such as $433 \mathrm{MHz}, 868 \mathrm{MHz}$ and 915 $\mathrm{MHz}$ which are in the sub-GHz frequency spectrum. This provide a better reliability against noise while drawing less energy than typical WiFi and Blue-tooth technology which operating at
$2.4 \mathrm{GHz}$ frequency bands. LoRa is regarded to have the best potential for recognition for supplying the LPWAN technology to numerous IoT applications.

LoRa is developed by Semtech using a single hop communication technology that is long range, low power and low bit rate. IoT applications is the purpose for it to be used for, where devices are battery powered and limited bandwidth. LoRa is a candidate technology for communication devices with low bandwidth industrial applications because of its scalability and price [4].

LoRa used the Chirp Spread Spectrum which is a restrictive spread spectrum technique. This technique used narrowband signal with the same power density as the wider frequency band. Hence, this makes LoRa safe against network attacks, more resilient to interference and more robust against jamming attacks. Additionally, this technique increases of the spread spectrum innovation and offers resilience to the frequency confuse between a transmitter and a receiver [5].

The chip rate is equivalent to the modified data transfer capacity (chip-per-second-per-Hertz) and can take estimations of 125, 250 or $500 \mathrm{kHz}$. In addition, the spreading factor (SF) for a LoRa connection might be differed relying upon the correspondence separate and sought on-broadcast appointment. Since the spreading codes for various SFs are orthogonal, the synchronous transmission in a similar frequency channel utilizing diverse SFs is conceivable. Impedance issues are alleviated by utilizing the forward mistake re-dressing codes in a blend with frequency hopping spread spectrum (FHSS). Altogether, ten channels with various data transfer capacities are accessible for LoRa in the EU 868-870 MHz ISM band [6].

A standard MAC layer and reporting on-the-air activation is used by LoRaWan in which it enables the node to join a current system [7]. These guidelines empower one-bounce interchanges scaling from $2 \mathrm{~km}$ in thick urban with buildings and moving objects environments to $30 \mathrm{~km}$ in rural area with nothing but trees. Furthermore, these will bring further outcomes for low-power, huge scale 
observing applications as the applications can be deployed to an extreme range.

Based on previous research papers, several authors have investigated on the performance of the LoRaWAN modulation protocol where the authors are focused on widely different subject such as the radio communication range. These researches were conducted since the release of the first specification for LoRaWAN [8]. First, the authors of [9] have presented their in-depth analysis of LoRa performance for an indoor IoT applications. There has also been a comparison between LoRaWAN and other unlicensed technologies. For instance, in [10] experimenting on the throughput of several technologies compared with LoRaWAN in terms of their packet error rate.

Comparable to past research on LoRaWAN performance, this paper was focused on experimenting the performance of LoRaWAN for an outdoor IoT application. This paper contributes on: (1) measuring the device received signal strength (RSSI). (2) performance analysis in terms of packet loss and Signal to Noise Ratio (SNR) between the transmitter and the receiver end of the system. The scope of this paper was specifically on the capabilities of LoRa delivering reliable connection for an outdoor IoT applications.

For informative readings, this paper has been organized properly with the Methodology of this research is explained in Section 2. In section 3, the experiment for this research is presented. Lastly, a concluding paragraph will close this paper in Section 4.

\section{Methodology}

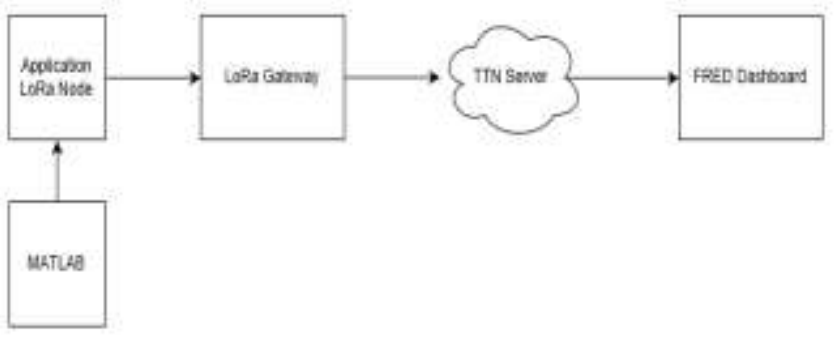

Fig. 2: System Block Diagram

A system was developed to emulate a various IoT applications using a LoRa Node. Based on Figure 2, the system used a set of data from MATLAB application. The data was retrieved from a recent Autonomous Vehicle project by UiTM. The data set contains the vehicle's distance, speed, steering angle, and its Global Positioning System (GPS) coordinate in terms of latitude and longitude. The system works by MATLAB sending the set of data to the ARDUINO Board. Then, the board that is connected to a DRAGI-NO LoRa Shield retrieved the data and convert it into a secured LoRaWAN protocol before sending it to a LoRa Gateway. From there, the gateway will then convert it again and send it further to FRED Dashboard.

\subsection{MATLAB Data}

\section{Load av data; \\ Setup serial communication with Arduino board; \\ Open serial port; \\ Select the first row of the data; \\ Select the first data in the row; \\ Send the data to Arduino via the serial port; \\ Periodically repeat the selected data with next in line;}

Fig. 3: Pseudo Code in MATLAB

By referring to Figure 3, the data set was first read in MATLAB application. By configuring a new set of command, the data was able to be push inside the Arduino Board. Serial Connection was used between the Arduino Board and MATLAB so that they can communicate with each other. The drawbacks by using this method, the data sent by the MATLAB cannot be view in Arduino Serial Monitor because it is using the same Serial Port with the USB which is Serial 0. However, the data can still be viewed by sending it back to the MATLAB.

\subsection{Arduino Board with DRAGINO LoRa Shield (LoRa Node)}

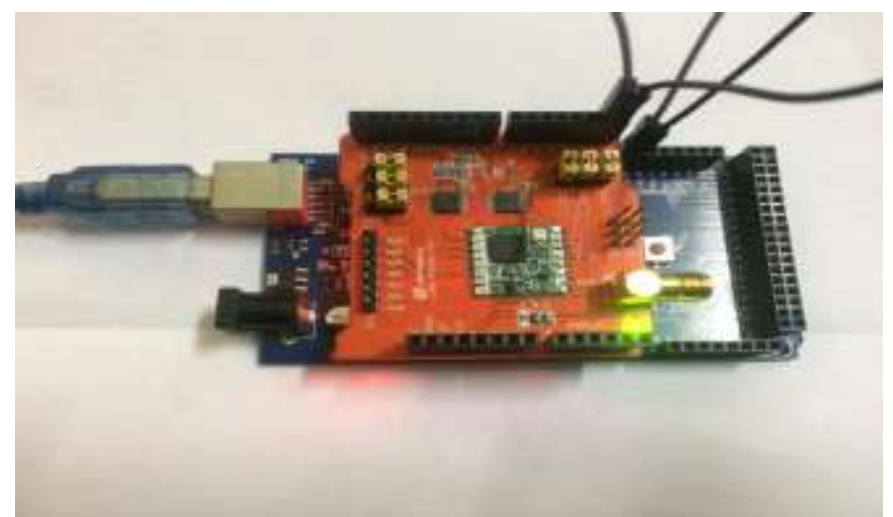

Fig. 4: DRAGINO Shield with Arduino MEGA 2560.

Figure 4 shows the boards used for the system. We were using the Arduino board used for this research is Arduino MEGA 2560, paired with DRAGINO LoRa Shield V1.4. The Arduino board will act as the application while the DRAGINO Shield will be the node connected to the gateway. Below is the figure of the Arduino MEGA 2560 connected to the Dragino LoRa Shield V1.4 or IoT applications.

The Dragino LoRa Shield is a long-range transceiver built to be able to use with Arduino with ease and based on open source library. The LoRa Shield uses a low data-rates which enables the user to send and receive data at long ranges. It provides ultra-long range spread spectrum communication and high interference immunity whilst minimizing current consumption. Using Semtech's patented LoRa modulation technique, the LoRa Shield can achieve a sensitivity of over $-148 \mathrm{dBm}$. Some of the specifications of the shield are $168 \mathrm{~dB}$ maximum link budget, $127 \mathrm{~dB}$ dynamic range RSSI and high sensitivity for as low $-148 \mathrm{dBm}$.

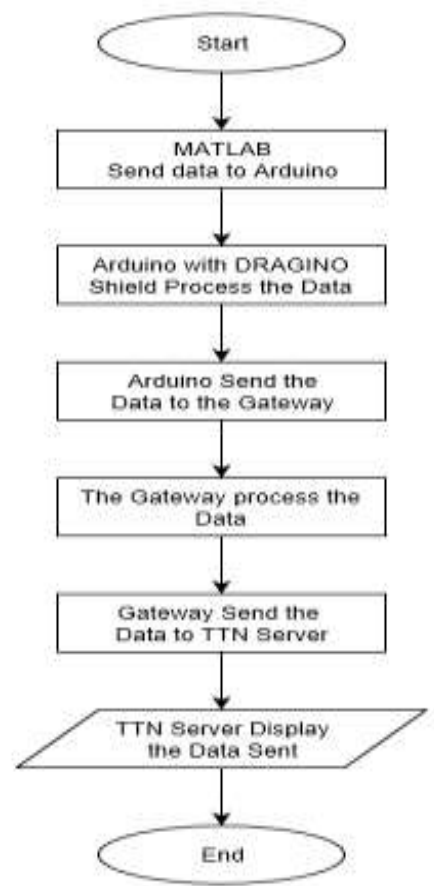

Fig. 5: Flow Chart of the End Device. 
Figure 4 shows the flowchart of the system that was developed. The data retrieved by the Arduino was sent to the LoRa gateway via the DRAGINO Shield. The data is being sent LoRaWAN modulation from the end device to the gateway. The time taken to complete the data transmission is being dependent on the coding rate of the DRAGINO Shield. The data retrieved by the gateway will be demodulated and forwarded to The Things Network (TTN) Server. Inside the TTN Server, the data is displayed according to the time the packets arrived. With user preference, the SNR and RSSI value can be viewed the server. This will ease the experiment for the performance of LoRa radio link

\subsection{Deployment}

The performance of LoRa radio link is impacted by the variety of physical layer parameters. Coding Rate (CR), Bandwidth (BW), and Spreading Factor (SF) are the three physical layer parameters, they will effectively be an influence on the bit rate of the modulation and the LoRa immunity to signal and noise interference

Table 1: Semtech SX1276 Receiver Sensitivity in dBM
\begin{tabular}{|c|c|c|c|c|c|c|}
\hline BW/SF & 7 & 8 & 9 & 10 & 11 & 12 \\
\hline 125 & -123 & -126 & -129 & -132 & -133 & -136 \\
\hline 250 & -120 & -123 & -125 & -128 & -130 & -133 \\
\hline 500 & -116 & -119 & -122 & -125 & -128 & -130 \\
\hline
\end{tabular}

Table 1 shows a summary of the LoRa receiver sensitivity with respect to Bandwidth (BW) and Spreading Factor (SF) reported in the data sheet. According to Table 1, the noise and receiver sensitivity will be determining by the LoRa radio channel bandwidth. By increasing the channel bandwidth, it expected that it will be resulting in the decrease of the receiver sensitivity. However, the increase in spreading factor will results in higher receiver's sensitivity. Hence, the sensitivity of the end device can be regulated with the settings of BW and SF. Besides, the packet delivery ratio will improve by the increasing of the coding rate.

Signal to Noise Ratio (SNR) as depicted in Figure 6 will approach the limit specified for the spreading factor (SF8) before the packet reception starts to fail. For an example, the SNR limit for spreading factor of 8 is $-10 \mathrm{~dB}$. If the transmitting packet are received at SNR of $-0 \mathrm{~dB}$, then the transmit power is reduced by 8 or $9 \mathrm{dBm}$, the reported SNR will have dropped to $-9 \mathrm{~dB}$. Hence, the packet reception will start to fail. The SNR values is a very good indication for approaching reception failure.

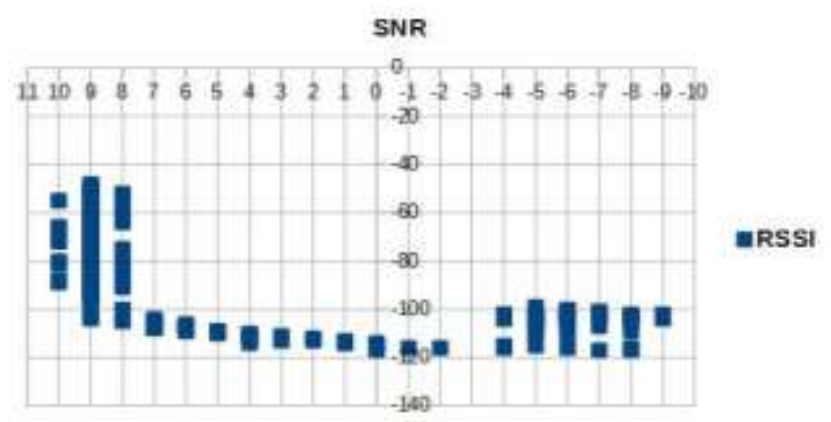

Fig. 6: SNR versus RSSI

The performance evaluation of LoRaWAN is conducted by setting up and register an application using The Things Network (TTN) server. TTN runs a web-based database that collect and store the transmitted packets from the LoRa end device. The TTN server provides an interface which provides information to the user such as the receiving signal strength indicator (RSSI) values and sequence number of the received packet.

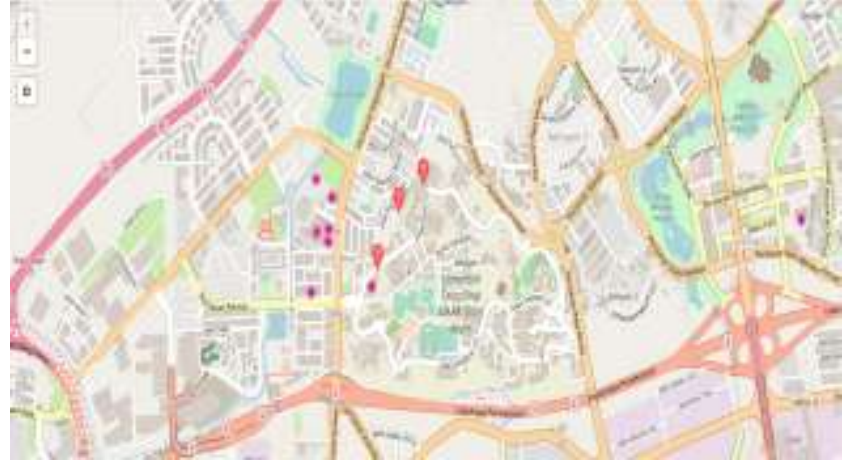

Fig. 7:. Map of current TTN Gateway.

Figure 7 shows the current available LoRa gateway in the nearest location to UiTM Shah Alam where the experiment is held. The experiment performed with end device was brought around the campus to take the reading. Experiment was performed during the weekdays to emulate the influence moving objects such as people movements and moving cars on the network performance.

For taking measurement of both Signal to Noise Ratio (SNR) and Received Signal Strength Indication Indicator (RSSI) values, by referring to Figure 8, the gateway is located at the green point on the map while the end device is located along the blue points. The location of the end device is set with $100 \mathrm{~m}$ apart starting from $100 \mathrm{~m}$ point to $500 \mathrm{~m}$. For each point, the end device transmits packet of data every several seconds. Hence, at every point of the end device, the result was recorded over the TTN server.

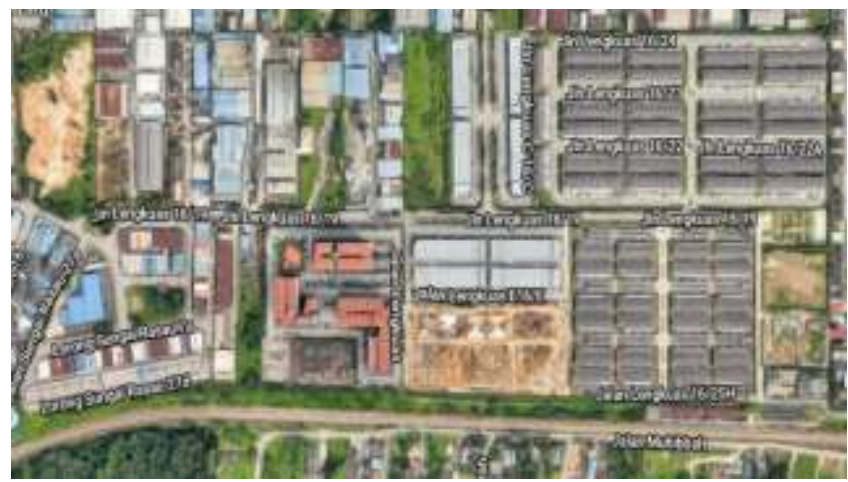

Fig. 8:. Map of the location of experiment.

\section{Results and Discussion}

\subsection{LoRa Outdoor Performance Analysis}

The general capability of LoRa radio receiver will be indicated with its Received Signal Strength Indicator (RSSI) values. The experiment was conducted with MATLAB sending out data to the application at various location with increasing of distances from the gateway, a constant SF of 12 and 19 bytes of payload for each data packets. Theoretically, based on Table 1, the minimum RSSI for every received packets will decrease as the distance between the gateway and end device increases.

Figure 9 and 10 show the recorded values of RSSI and SNR respectively. In Figure 9, the minimum value of RSSI measured at different location with the increased distances of $100 \mathrm{~m}$ from the gateway to the end device. The RSSI values decreases as the number of obstruction and distance to the gateway increases. However, the link between the end device and the gateway become more stable as the distance reach $200 \mathrm{~m}$ onwards with the maximum change of only 4 between subsequent 7 locations. Next, in Figure 10 , the values of recorded SNR for each point with end device periodically transmit the data is shown. Here, the SNR values decreases from $100 \mathrm{~m}$ marks onward. However, correspond to the 
RSSI values, after $100 \mathrm{~m}$ point to $200 \mathrm{~m}$ point, the values of the SNR also become more stable as the distance increases.

Table 1: LoRa Radio Parameter Settings

\begin{tabular}{|c|c|c|}
\multicolumn{3}{|c|}{ LoRa Settings } \\
\hline Parameter & Value & Details \\
\hline Frequency & Asia $923-925 \mathrm{MHz}$ & Class A \\
\hline Bandwidth (BW) & $125 \mathrm{kHz}$ & $\begin{array}{c}\text { Default LoRaWAN } \\
\text { Configuration }\end{array}$ \\
\hline $\begin{array}{c}\text { Spreading } \\
\text { Factor (SF) }\end{array}$ & $7,8,9,10,11,12$ & $\begin{array}{c}\text { Selected LoRaWAN } \\
\text { Configuration }\end{array}$ \\
\hline Tx Payload Size & 19 bytes & Used with SF \\
\hline
\end{tabular}

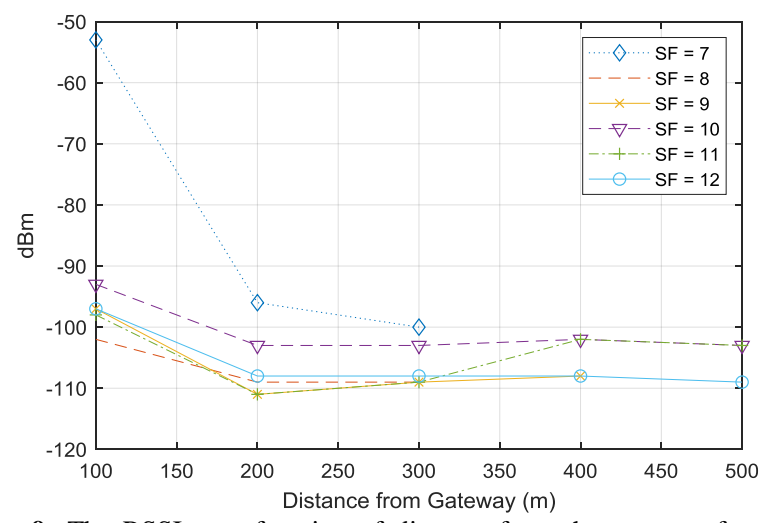

Fig. 9: The RSSI as a function of distance from the gateway for ever Spreading Factor used in the experiment

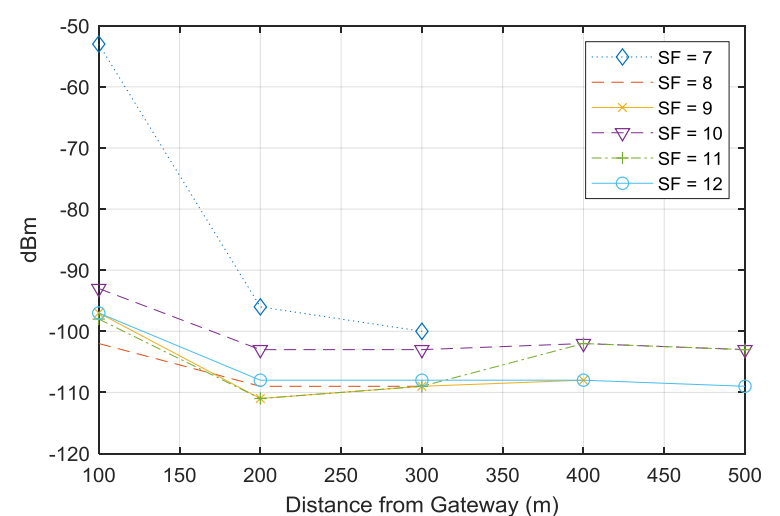

Fig. 10: The SNR as a function of distance from the gateway for ever Spreading Factor used in the experiment

The reason for both RSSI and SNR values to become stable at a higher distance is related to the use of higher SF value. High value of SF enables the packet receiving at the gateway to be (1) synchronize in time, (2) adjusting the gain of the receiver amplifier. The theory behind this is the data packet sent with the higher value of SF will be received with specific settings. However, with the multiple signal path that presents within the room where the gateway is located, the signal strength will change rapidly. For that reasons, it is safe to say that both RSSI and SNR values are changing with respect to the SF and distance in particular. Hence, a hypothesis can be made where, the further the distance between the end device and the gateway, the lower values of RSSI and SNR.

\subsection{UiTM AV1 Dashboard Implementation}

MATLAB variables in multidimensional array in the main computer consisting of UiTM Av1 status such as GPS coordinates, speed, orientation, and distance travelled are converted to JSON messages and transmitted via LoRa LPWAN to MQTT broker (e.g. Eclipse Mosquito) installed in a secondary computer. Using node-RED, the secondary computer is also configured as a MQTT client and listens to the default TCP port number, e.g. port 1883. Any received JSON message is split into several variables and organized using several message processing nodes (i.e. composed as flow in node-
RED), in order to insert the variables into a time series database named InfluxDB, the message payload is formatted and structured to include the measurement, field and tag which follow the schema set earlier in the database. The secondary computer is a Microsoft Surface Pro which acts as a dashboard for the UiTM AV1. The function of the dashboard is to display and visualize the metrics collection using Grafana, integrated with InfluxDB as the data source. The final dashboard for UiTM AV1 is shown in Figure 11.

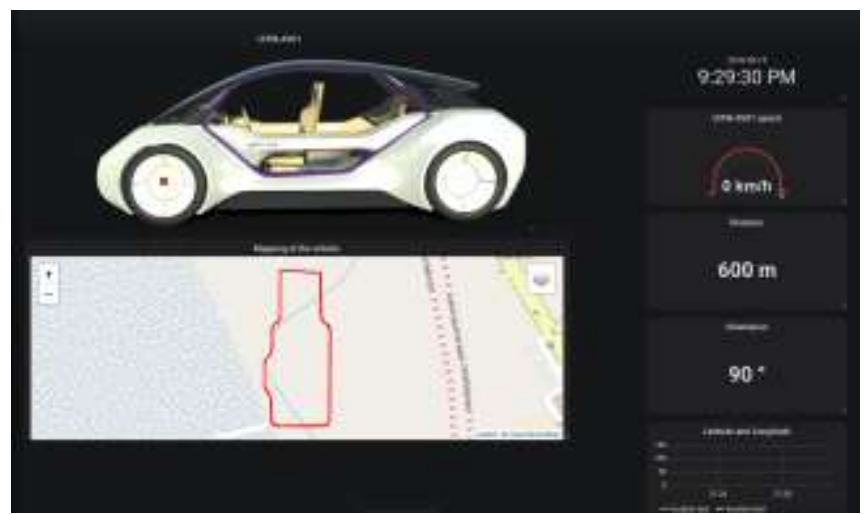

Fig. 11: The Dashboard for UiTM Autonomous Vehicle 1 (UiTM AV1)

\section{Conclusion}

In conclusion, the performance analysis for LoRa IoT applications is successfully done. This project gives an insight on how the LoRa technology can provide a better solution for IoT platforms. It will also enable people to decide whether to use LoRa for their side projects or even using it as their school assignment projects. Lastly, this project can also be a reference to future researcher that want to use LoRa for their research.

\section{Acknowledgement}

This research is funded by Institute of Research Management and Innovation (IRMI), Universiti Teknologi MARA (UiTM), Shah Alam, Selangor, Malaysia under the Research Grant Scheme No: 600-IRMI/DANA 5/3/ARAS (0024/2016). The authors also gratefully acknowledge Faculty of Electrical Engineering UiTM Shah Alam for all the support given during this research.

\section{References}

[1] Wortmann, F., and Flüchter, K. (2015). Internet of things. Business and Information Systems Engineering, 57(3), 221-224.

[2] S. D. T. Kelly, N. K. Suyadevara and S. C. Mukhopadhyay, "Towards the implementation of IoT for environmental condition monitoring in homes", IEEE Sensors Journal, 13(10), 3846-3853, 2013.

[3] M. Centenaro, L. Vangelista, A. Zanella, and M. Zorzi. "Longrange communications in unlicensed bands: The rising stars in the iot and smart city scenarios", IEEE Wireless Communications, 23(5), 60-67, 2016.

[4] J. Haxhibeqiri, A. Karaagac, F. V. den Abeele, W. Joseph, I. Moerman, J. Hoebeke, "LoRa indoor coverage and performance in an industrial environment: Case study", Proceedings of the 22nd IEEE International Conference on Emerging Technologies And Factory Automation, pp. 1-8, 2017.

[5] U. Raza, P. Kulkarni, and M. Sooriyabandara, "Low power wide area networks: An overview", IEEE Communications Surveys and Tutorials, 19(2), 855-873.

[6] J. Petäjäjärvi, K. Mikhaylov, A. Roivainen, T. Hänninen, and M. Pettissalo, "On the coverage of LPWANs: Range evaluation and channel attenuation model for LoRa technology", Proceedings of the 14th International Conferences on ITS Telecommunications, pp. 55-59, 2016.

[7] K. Mikhaylov, J. Petäjäjärvi, J. Haapola, and A. Pouttu, "D2D communications in LoRaWAN low power wide area network: 
From idea to empirical validation", Proceedings of the IEEE International Conference on Communications Workshops, pp. 2-7, 2017.

[8] LoRa Alliance, "LoRa Alliance-wide area networks for IoT," https://www. lora-alliance. org/.

[9] E. D. Ayele, C. Hakkenberg, J. P. Meijers, K. Zhang, N. Meratnia and P. J. M. Havinga, "Performance analysis of LoRa radio for an indoor IoT application", Proceedings of the IEEE International Conference on Internet of Things for the Global Community, pp. 16, 2017.

[10] B. Reynders, W. Meert, and S. Pollin. "Range and coexistence analysis of long range unlicensed communication", Proceedings of the 23rd International Conference on IEEE Telecommunications, pp. $1-6,2016$

[11] Semtech, "Semtech

SX1276", https://www.semtech.com/products/wireless-rf/loratransceivers/SX1276.

[12] N. Vatcharatiansakul, P. Tuwanut and C. Pornavalai, "Experimental performance evaluation of LoRaWAN: A case study in Bangkok", Proceedings of the IEEE 14th International Joint Conference on Computer Science and Software Engineering, pp. 14, 2017. 